\title{
(- OPEN ACCESS \\ Multidrug-resistant tuberculosis (MDR-TB) and multidrug-resistant HIV (MDR-HIV) syndemic: challenges in resource limited setting
}

\author{
Christian Francisco, ${ }^{1}$ Mary Ann Lansang, ${ }^{1}$ Edsel Maurice Salvana, ${ }^{1}$ Katerina Leyritana ${ }^{2}$
}

'Section of Infectious Diseases, Department of Medicine, Philippine General Hospital, Manila, Philippines

${ }^{2}$ Sustained Health Initiatives of the Philippines, Mandaluyong, Philippines

Correspondence to Dr Christian Francisco, christianfrancisco1@gmail.com

Accepted 4 August 2019

Check for updates

(c) BMJ Publishing Group Limited 2019. Re-use permitted under CC BY-NC. No commercial re-use. See rights and permissions. Published by BMJ.

To cite: Francisco $C$ Lansang MA, Salvana EM, et al. BMJ Case Rep

2019:12:e230628

doi:10.1136/bcr-2019

230628

\section{SUMMARY}

Tuberculosis (TB) is common among persons living with HIV. This public health concern is aggravated by infection with multidrug-resistant organisms and adverse effects of polypharmacy. There are few published cases of multidrug-resistant tuberculosis (MDR-TB) in multidrug-resistant HIV (MDR-HIV) infected patients. We report a case of a 29-year-old Filipino man with HIV on zidovudine (AZT)-containing antiretroviral therapy (ART) but was eventually shifted to tenofovir due to anaemia. He presented with left flank tenderness, which was found to be due to an MDR-TB psoas abscess, and for which second-line anti-TB treatment was started. HIV genotyping showed MDR-HIV infection susceptible only to AZT, protease inhibitors and integrase inhibitors. Subsequently, he developed neck abscess that grew Mycobacterium avium complex and was treated with ethambutol and azithromycin. ART regimen was revised to AZT plus lamivudine and lopinavir/ritonavir. Erythropoietin was administered for recurrent AZTinduced anaemia. Both abscesses resolved and no recurrence of anaemia was noted.

\section{BACKGROUND}

The tuberculosis (TB) and HIV coinfection is a global syndemic that increases morbidity and mortality from both diseases. It jeopardises disease control efforts, especially in developing countries like the Philippines. ${ }^{1}$ Complicating the scenario is the emergence of drug resistance in both disease entities. Despite the advent of new diagnostic tests to detect resistance, there have been only few published cases of multidrug-resistant tuberculosis (MDR-TB) and multidrug-resistant HIV (MDR-HIV) coinfection. ${ }^{2}$ Additional challenges faced are infection with other non-tuberculous mycobacteria (NTM) and unavailability of other classes of antiretroviral therapy (ART) drugs in resource-limited settings. Triple coinfection (TB, HIV, NTM) complicates clinical management with regard to antimycobacterial and ART choices, adverse drug events and compliance issues. We report a complicated case of multiple coinfections and the dilemmas encountered in the course of management.

\section{CASE PRESENTATION}

A 29-year-old Filipino man with HIV presented with fever and left flank tenderness. He was diagnosed with HIV infection 1 year prior to his symptoms with a baseline CD4 count of 246 cells/ $\mathrm{mm}^{3}$. Workup for active TB which included chest radiographs, direct sputum smear microscopy for acid-fast bacilli (AFB) and sputum GeneXpert MTB/ Rif were all negative. Tuberculin skin test (TST) to screen for latent TB infection (LTBI) was not done. We started him on isoniazid preventive therapy (IPT), consisting of isoniazid $300 \mathrm{mg}$ tablet once per day for 6 months. On diagnosis, we started him on an ART regimen consisting of lamivudine (3TC), zidovudine (AZT) and nevirapine (NVP). Baseline HIV genotyping test is not routinely performed in our setting. Baseline and repeat HIV RNA viral load after 2 months of ART initiation were not done as it is not part of local treatment guidelines. Six months after ART initiation, he developed AZT-induced anaemia. We discontinued AZT and shifted to tenofovir (TDF).

One year after HIV diagnosis, the patient experienced persistent low back pain with radiation to the left flank area, associated with high-grade fevers. A working diagnosis of a psoas abscess was made. $\mathrm{He}$ had no previous history and treatment for TB and other opportunistic infections. He was subsequently admitted for further workup.

\section{INVESTIGATIONS}

On admission, ultrasound revealed a fluid collection in the left psoas area. Whole abdominal CT scan confirmed the diagnosis, showing a left paravertebral hypodense collection in the left psoas muscle, consistent with abscess formation (figure 1). A CT-guided aspiration biopsy was done. Microbiological workup of the abscess fluid showed no organism on Gram stain but showed AFB on ZiehlNeelsen stain. Xpert MTB/Rif detected Mycobacterium tuberculosis (MTB) with rifampicin resistance. Routine bacterial culture did not grow any organisms. Mycobacterial culture (BACTEC 460 TB system, Beckton Dickinson, Maryland, USA) with drug-susceptibility testing (DST) eventually grew MTB, resistant to all first-line anti-TB drugs (including rifampicin, isoniazid, pyrazinamide, ethambutol and streptomycin).

Six months after the TB diagnosis, he developed a left lateral neck mass. Ultrasound revealed an ill-defined hypoechoic focus measuring $4.6 \times 5.5 \times 4.8 \mathrm{~cm}$ with an approximate volume of $83 \mathrm{~mL}$. CT scan confirmed the neck abscess (figure 2). Aspiration was done. Gram stain showed 


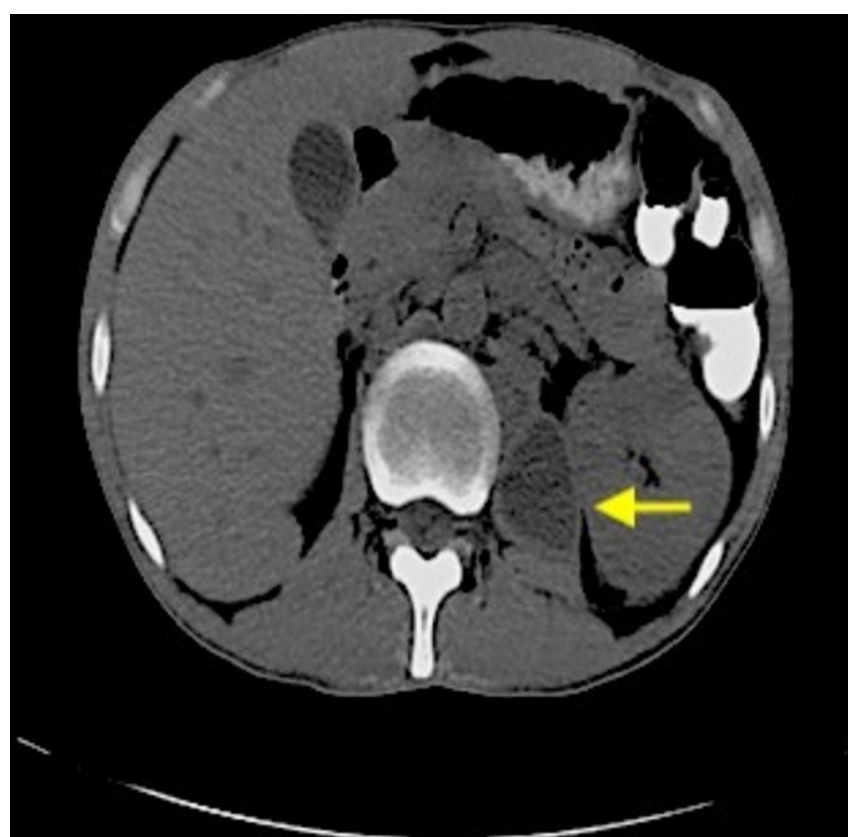

Figure 1 A contrast-enhanced abdominal CT scan showing a $12.8 \times 2.9 \times 3.5 \mathrm{~cm}$ left psoas abscess (pointed by the yellow arrow).

5-10 polymorphonuclear cells/high power field (hpf) and 0-1 cocci in pairs/hpf. The Ziehl-Neelsen stain showed the presence of AFB. No pathogens were isolated on bacterial culture, while the mycobacterial culture grew Mycobacterium avium after 11 days of incubation. However, DST for M. avium was not done due to limited patient funds.

Due to the occurrence of these opportunistic infections, CD4 count was repeated, showing a decrease from 246 to 37 cells/ $\mathrm{mm}^{3}$ despite good self-reported adherence to ART $(3 \mathrm{TC}+\mathrm{N}$ $\mathrm{VP}+\mathrm{TDF})$. HIV-1 genotyping was done, revealing circulating recombinant form 01_AE (CRF01_AE) subtype. K101E, Y181C and Y181I/V mutations were found conferring resistance to all non-nucleoside reverse transcriptase inhibitors (NNRTIs); as well as $\mathrm{M} 184 \mathrm{~V}$ and $\mathrm{K} 65 \mathrm{R}$ mutations indicating resistance to all locally available nucleoside reverse transcriptase inhibitors (NRTIs) except AZT. The virus remained fully susceptible to

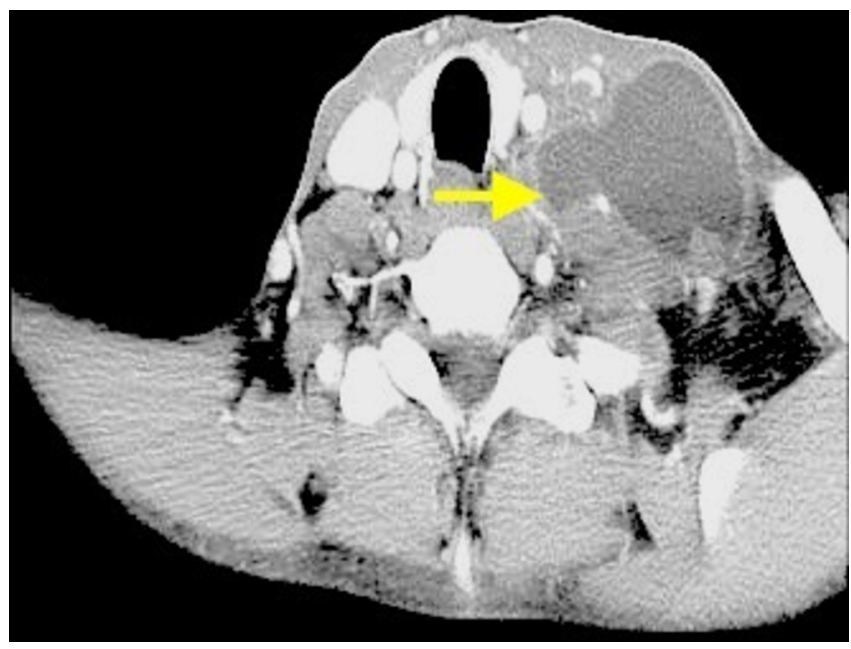

Figure 2 A contrast enhanced neck CT scan showing a $4.6 \times 5.5 \times 4.8 \mathrm{~cm}$ abscess (pointed by the yellow arrow) with an approximate volume of $83 \mathrm{cc}$ on the left lateral neck. protease inhibitors (PIs) and integrase strand transfer inhibitors (INSTIs).

\section{TREATMENT}

Following confirmation of the susceptibility of the MDR-TB psoas abscess, the patient was started on the following regimen: levofloxacin $750 \mathrm{mg}$ tablet once per day; cycloserine $200 \mathrm{mg}$ tablet, 2 tablets once per day; prothionamide $250 \mathrm{mg} / \mathrm{tablet}, 3$ tablets once per day; and kanamycin $750 \mathrm{mg}$ intramuscularly per day. The patient experienced adverse drug reactions from the regimen, including occasional nausea, vomiting and dizziness, for which metoclopramide was given. We managed the $M$. avium neck abscess with azithromycin $500 \mathrm{mg}$ tablet every other day and ethambutol $400 \mathrm{mg}$ tablet, 2 tablets per day, for 1 year.

Based on the patient's HIV genotyping test, the virus was resistant to all NNRTIs and NRTIs except AZT and remained susceptible to all PIs and INSTIs. However, due to the unavailability of INSTIs in our setting, we shifted back ART to 3TC-AZT-based regimen and the addition of ritonavir-boosted lopinavir (LPV/r). After a few months on the new ART regimen, AZT-induced anaemia ensued, necessitating repeated blood transfusions. We then provided the patient with ferrous sulfate $325 \mathrm{mg}$ tablet once per day and erythropoietin (EPO) alpha 4000 units subcutaneously given two times per day to prevent anaemia recurrence.

\section{OUTCOME AND FOLLOW-UP}

Despite the adverse effects from antimycobacterial medications and ART, the patient was able to complete treatment for $M$. avium for 12 months and MDR-TB treatment for 24 months. Neck and abdominal CT scan after treatment revealed resolution of the abscesses. There was no recurrence of anaemia while on EPO and ferrous sulfate. His most recent CD4 count was 778 cells $/ \mathrm{mm}^{3}$ and HIV viral load was 192 copies $/ \mathrm{mL}$.

\section{DISCUSSION}

TB-HIV coinfection is a public health threat. Prevalence of MDR-TB among HIV-infected individuals is highly variable, ranging from $0.6 \%$ to $76 \%,{ }^{3}{ }^{4}$ and this coinfection is associated with higher mortality and morbidity. ${ }^{3}$ However, very few have reported on the outcomes of patients with MDR-TB and MDR-HIV coinfection. ${ }^{2}$ This patient is one of a handful of documented cases of MDR-TB and HIV coinfection, and its management revealed some therapeutic challenges.

Current guidelines recommend that ART be started in all persons living with HIV (PLHIV) regardless of CD4 count. ${ }^{5}$ However, ART can cause a range of adverse reactions that may be acute and life-threatening or chronic and insidious. ${ }^{6}$ Our patient developed AZT-induced anaemia a few months after starting ART. This occurs in $14.6 \%$ of patients on AZT-containing regimen. ${ }^{7}$ Management includes shifting to another NRTI, such as TDF. Anaemia usually resolves within 2 weeks after discontinuation of AZT.

HIV infection is the leading risk factor for the progression of LTBI to active disease. ${ }^{9}$ Initiation of IPT is recommended after ruling out active TB. In our patient, active TB was ruled out after an unremarkable chest radiograph, negative bacteriological tests for TB and absence of constitutional symptoms. A meta-analysis has shown that the absence of cough, weight loss, fever and night sweats has a negative predictive value of $98 \%$ against the diagnosis of active TB. ${ }^{10}$ TST is not required for initiating IPT among PLHIV as recommended by WHO. ${ }^{11}$ It is also important to note that NTM infections and previous bacillus CalmetteGuérin vaccination may cause false-positive TST among the 
HIV-infected population but this should not prevent IPT initiation. ${ }^{12}$ In addition, the sensitivity of TST for detecting LTBI is reduced for PLHIV. ${ }^{13}$ Despite the recommendations to use a lower cut-off values for positive TST result for PLHIV $(5 \mathrm{~mm}$ diameter instead of $10 \mathrm{~mm}$ diameter induration), ${ }^{14}$ interpretation of the TST remains to be difficult since skin test anergy is common among PLHIV and is associated with low CD4 count. ${ }^{13}$

Fever in PLHIV is associated with an extensive list of infectious and non-infectious aetiologies. In settings where TB-HIV coinfection is common, TB should always be considered and prompt workup should be instituted. ${ }^{15}$ In this case report, our patient presented with an indolent fever, constitutional symptoms and low back pain, which was caused by a tuberculous psoas abscess. TB psoas abscess formation is not unusual among PLHIV. ${ }^{16} 17$ Its management involves aspiration of the abscess and starting appropriate anti-TB drugs. In our case, second-line TB drugs were initiated due to the presence of AFB in the abscess fluid, positive Xpert MTB/Rif (MTB detected/rifampicin resistance detected), and DST. Some studies have shown that the presence of rifampicin resistance is a marker of MDR-TB in areas with high burden of infection. ${ }^{18}$ However, Xpert MTB/Rif may not be an optimal stand-alone test for the diagnosis of extrapulmonary TB and should be interpreted with caution. Mycobacterial culture with DST should be included in the workup of an HIV-infected patient suspected to have TB for the following reasons: higher prevalence of MDR-TB among HIV-infected, NTM coinfections, and serve as guidance in the targeted management of MDR-TB. ${ }^{19} 20$

NTM infection, particularly Mycobacterium avium complex (MAC), is seen in $2 \%$ of HIV-infected patients ${ }^{21} 22$ especially among those with CD 4 count $<50$ cells $/ \mathrm{mm}^{3}$. Various case reports have discussed MTB and NTM coinfection among patients with HIV, and there are even reports of coexisting MTB-NTM in a single abscess site such as the brain ${ }^{19}$ and lymph nodes. ${ }^{23}$ Treatment of NTM is commonly empiric since most of the existing drugs were not specifically developed for the treatment of NTM. Drug activities are usually extrapolated from MTB treatment. ${ }^{24}$ Azithromycin and ethambutol were added to this patient's MTB treatment regimen to cover for MAC. Randomised controlled trials have shown efficacy of azalides (azithromycin or clarithromycin) combined with ethambutol in treating disseminated MAC. ${ }^{25}{ }^{26}$ Susceptibility studies have shown that azithromycin and ethambutol remain a good option for MAC treatment. However, it is still recommended that drug susceptibility test be performed for all known NTM isolates. ${ }^{27}$

Drug-resistance testing (DRT) through HIV genotyping detects clinically significant mutations in the HIV genome, and is a useful tool for assessing resistance among the commonly used ART drugs, particularly NRTIs and NNRTIs. ${ }^{28}$ The downward trend in our patient's CD4 count and the occurrence of opportunistic infections raised the suspicion of possible resistance. Although not routinely done in resource-limited settings, HIV viral load and (if not suppressed) genotyping is recommended in a patient on ARTs who develops new opportunistic infections, a protracted infection course despite appropriate treatment, or recurrence of a previously resolved infection. However, the utilisation of this test on a larger scale is limited by its availability, accessibility and relatively high cost in resource-constrained settings. Underutilisation of DRT may lead to inadequate investigation of HIV resistance and under-reporting of cases. This may be the reason behind the dearth of published cases of MDR-TB/ MDR-HIV coinfection.

The greatest challenge in the management of this case was weighing the HIV genotyping result against the risk of recurrence of a documented adverse drug reaction. In our setting, ART is currently limited to NRTI (3TC, AZT), NNRTI (EFV, NVP) and PI (LPV/r). The current Philippine HIV epidemic is driven by the predominance of the CRF01_AE subtype ${ }^{29} 30$ in contrast to the predominance of subtype $\bar{B}$ in the Americas, Western Europe and Australasia, ${ }^{31}$ and subtype $\mathrm{C}$ in Africa. ${ }^{31}$ Infection with CRF01_AE has been associated with rapid progression to AIDS and possibly higher mortality, ${ }^{31} 32$ thus ART classes that rapidly suppress HIV viral load are essential. INSTIs comprise a key drug class that has been shown in randomised controlled trials to be more effective in rapidly decreasing HIV viral load within 12 weeks when compared with the standard NNRTI and NRTI combinations, ${ }^{33}$ and this effect is similar across most INSTIs. ${ }^{33}$ Reports have shown an increasing numbers of pretreatment NNRTI resistance among ART-naïve patients ${ }^{35-37}$ and can be $>25 \%$ among those experiencing ART failure..$^{38}$ In our case, HIV genotyping revealed mutations in K101E and Y181C, causing resistance to EFV and $\mathrm{Y} 181 \mathrm{I} / \mathrm{V}$ mutations causing resistance to NVP, etravirine and rilpivirine. These transmitted drug resistance (TDR) patterns were reported to occur in $2.2 \%$ in a patient cohort in Malawi. ${ }^{35}$ On the other hand, NRTI mutations are less prevalent, ranging from $1.6 \% \%^{38}$ to $4 \%{ }^{39}$ among ART-experienced and ART-naïve, respectively. In the Philippines, overall HIV-1 TDR by conventional Sanger-based sequencing is 9.7\% overall (NNRTI: 6.2\%, NRTI: $1.8 \%$ and PI: $2.7 \%$ ) and was even higher on next-generation sequencing at $5 \%$ minority variant cut-off (19.5\% overall, NNRTI: $13.3 \%$, NRTI: $2.7 \%$, PI: $4.4 \%$, INSTI $1.8 \%) .^{40}$ In our case, the patient had M184 mutations conferring resistance to 3TC, emtricitabine (FTC), didanosine (ddI) and abacavir (ABC), while K65R causes intermediate to high-level phenotypic and clinical resistance to TDF, $\mathrm{ddI}, \mathrm{ABC}$ and $\mathrm{d} 4 \mathrm{~T}$ and low level to intermediate phenotypic and

\section{Learning points}

- The rarity and complexity of multidrug-resistant tuberculosis and multidrug-resistant HIV coinfection pose a great challenge to all healthcare professionals providing care to persons living with HIV.

- The rule of parsimony is usually the basis of good clinical judgement, but this case showed that opportunistic infections can coexist in the setting of HIV infection.

- With the increased use of molecular diagnostic methods, appropriate diagnostic tests should be requested and interpreted with caution, especially when dealing with multidrug-resistant organisms.

- In resource-limited settings, the choice of antiretroviral therapy (ART) drugs is limited to older classes of nucleoside reverse transcriptase inhibitor, non- nucleoside reverse transcriptase inhibitor (NNRTI) and protease inhibitor that are associated with many adverse effects. Physicians should be familiar with the pharmacokinetics of ART drugs, their expected adverse effects and possible interactions with other drugs.

- In the setting of increasing HIV resistance to standard ART recommended by the WHO, integrase strand transfer inhibitors (INSTIS) are preferred and should be made widely available given their better tolerability profile and their ability to rapidly reduce viral load.

- In the absence of INSTIs, baseline drug-resistance testing should be performed due to increasing transmitted drug resistance, particularly to NNRTIs. 
clinical resistance to 3TC and FTC. Drug resistance surveillance of CRF01_AE subtype in a Chinese cohort experiencing ART failure reported occurrence of $\mathrm{M} 184 \mathrm{~V}$ and $\mathrm{K} 65 \mathrm{R}$ mutations at $64.98 \%$ and $15.97 \%$, respectively, among treatment failures. ${ }^{38}$ The M184 mutation is not a contraindication to a continued treatment with 3TC or FTC, thus 3TC remained a part of our patient's ART regimen. Studies have shown that the presence of M184V mutation is not associated with virological failure even if 3 TC or FTC were continued, as long as other active drugs are present. $^{41}$

In our setting where new ART classes remain unavailable, there was a risk in resuming AZT, which previously caused profound anaemia in our patient. Weighing the benefit over the risk, control of the HIV infection was paramount, and the anaemia was managed through blood transfusions as needed. AZT-induced anaemia is postulated to occur due to low levels of endogenous EPO, unresponsiveness to the usual endogenous concentrations, or compounded by HIV infection. ${ }^{42}$ EPO administration in patients with AZT-induced anaemia has been showed to improve mean haematocrit levels and decrease frequency of blood transfusions. ${ }^{43}$ PIs remain the preferred second-line ART in resource limited setting. ${ }^{5}$ Baseline DRT should be routinely performed as NNRTI resistance increases, and if not done, PIs should be considered as part of the initial ART regimen due to its high genetic barrier for resistance ${ }^{44}$ However, PIs are associated with metabolic complications including dyslipidemia, hyperglycemia and lipodystrophy. ${ }^{45}$ INSTIs may be a better option since they are potent, well tolerated and have less adverse effects. ${ }^{46}$

Acknowledgements The authors would like to thank their colleagues Dr Marie Joyce Santos, Dr Maria Jonelyn Colobong, and the clinic staff of SAGIP Unit of the Philippine General Hospital and Sustained Health Initiatives of the Philippines.

Contributors CF, MAL and EMS conceived the design of the study, performed data collection and analysis, revised the manuscript and approved the final manuscript. $\mathrm{KL}$ performed data collection and analysis, revised the manuscript and approved the final manuscript.

Funding The authors have not declared a specific grant for this research from any funding agency in the public, commercial or not-for-profit sectors.

Competing interests None declared.

Patient consent for publication Obtained.

Provenance and peer review Not commissioned; externally peer reviewed.

Open access This is an open access article distributed in accordance with the Creative Commons Attribution Non Commercial (CC BY-NC 4.0) license, which permits others to distribute, remix, adapt, build upon this work non-commercially, and license their derivative works on different terms, provided the original work is properly cited and the use is non-commercial. See: http://creativecommons.org/ licenses/by-nc/4.0/

\section{REFERENCES}

1 Pepper DJ, Schomaker M, Wilkinson RJ, et al. Independent predictors of tuberculosis mortality in a high HIV prevalence setting: a retrospective cohort study. AIDS Res Ther 2015;6:35.

2 Satti H, McLaughlin MM, Seung KJ. Drug-resistant tuberculosis treatment complicated by antiretroviral resistance in HIV coinfected patients: a report of six cases in Lesotho. Am J Trop Med Hyg 2013;89:174-7.

3 Hurtado RM, Meressa D, Goldfeld AE. Treatment of drug-resistant tuberculosis among people living with HIV. Curr Opin HIV AIDS 2018:13:478-85.

4 Zürcher K, Ballif M, Fenner L, et al. Drug susceptibility testing and mortality in patients treated for tuberculosis in high-burden countries: a multicentre cohort study. Lancet Infect Dis 2019;19:298-307.

5 World Health Organization. Guideline on When to Start Antiretroviral Therapy and on Pre-exposure Prophylaxis for HIV. 2015. http://apps.who.int/iris/bitstream/10665/ 186275/1/9789241509565_eng.pdf

6 AIDSinfo. Guidelines for the Use of antiretroviral agents in HIV-1-infected adults and adolescents. http://aidsinfo.nih.gov/guidelines

7 Dash KR, Meher LK, Hui PK, et al. High incidence of zidovudine induced anaemia in HIV infected patients in Southern Odisha. Indian J Hematol Blood Transfus 2015;31:247-50.
8 Max B, Sherer R. Management of the adverse effects of antiretroviral therapy and medication adherence. Clin Infect Dis 2000:30:S96-S116.

9 van der Sande MA, Schim van der Loeff MF, Bennett RC, et al. Incidence of tuberculosis and survival after its diagnosis in patients infected with HIV-1 and HIV-2. AIDS 2004; 18:18

10 Getahun H, Kittikraisak W, Heilig CM, et al. Development of a standardized screening rule for tuberculosis in people living with HIV in resource-constrained settings: individual participant data meta-analysis of observational studies. PLoS Med 2011;8:e1000391.

11 World Health Organization. Guidelines for intensified case finding and isoniazid prevention therapy for people living with HIV in resource constrained settings. Geneva: World Health Organization, 2011.

12 Centers For Disease Control And Prevention. Guidelines for prevention and treatment of opportunistic infections in HIV-infected adults and adolescents: recommendations from Centers For Disease Control And Prevention, the National Institutes of Health, and the HIV Medicine Association of the Infectious Diseases Society of America. MMWR Recomm Rep 2009:58:1.

13 Cobelens FG, Egwaga SM, van Ginkel T, et al. Tuberculin skin testing in patients with HIV infection: limited benefit of reduced cutoff values. Clin Infect Dis 2006:43:634-9.

14 Long R, Houston S, Hershfield E, et al. Recommendations for screening and prevention of tuberculosis in patients with HIV and for screening for HIV in patients with tuberculosis and their contacts. CMAJ 2003;169:789-91.

15 Trinh QM, Nguyen HL, Nguyen VN, et al. Tuberculosis and HIV co-infection-focus on the Asia-Pacific region. Int J Infect Dis 2015;32:170-8.

16 Kozakis L, Ballachandran T. Bilateral psoas abscesses in an HIV-positive patient. Int STD AIDS 2004:15:841-3.

17 Rakesh TP, Kidangazhiathmana A, Jyothish K, et al. Large Primary Tuberculous Psoas Abscess in an HIV patient - A Case report. SAARC Journal of Tuberculosis, Lung Diseases \& HIV/AIDS 2014.

18 Denkinger CM, Pai M, Dowdy DW, et al. Do we need to detect isoniazid resistance in addition to rifampicin resistance in diagnostic tests for tuberculosis? PLoS One 2014;9:e84197.

19 Karne SS, Sangle SA, Kiyawat DS, et al. Mycobacterium avium-intracellulare brain abscess in HIV-positive patient. Ann Indian Acad Neurol 2012;15:54-5.

20 Isaakidis P, Das M, Kumar AM, Ajay M, et al. Alarming levels of drug-resistant tuberculosis in HIV-infected patients in metropolitan Mumbai, India. PLoS One 2014;9:e110461

21 McCarthy KD, Cain KP, Winthrop KL, et al. Nontuberculous mycobacterial disease in patients with HIV in Southeast Asia. Am J Respir Crit Care Med 2012:185:981-8.

22 Nyamogoba HD, Mbuthia G, Mining S, et al. HIV co-infection with tuberculous and non-tuberculous mycobacteria in western Kenya: challenges in the diagnosis and management. Afr Health Sci 2012;12:305-11.

23 Sharma K, Mewara A, Gupta N, et al. Multiplex PCR in diagnosis of M. tuberculosis and M. avium co-infection from lymph node in an AIDS patient. Indian J Med Microbiol 2015;33:151-3

24 Egelund EF, Fennelly KP, Peloquin CA. Medications and monitoring in nontuberculous mycobacteria infections. Clin Chest Med 2015;36:55-66.

25 Dunne M, Fessel J, Kumar $\mathrm{P}$, et al. A randomized, double-blind trial comparing azithromycin and clarithromycin in the treatment of disseminated Mycobacterium avium infection in patients with human immunodeficiency virus. Clin Infect Dis 2000;31:1245-52.

26 Ward TT, Rimland D, Kauffman C, et al. Randomized, open-label trial of azithromycin plus ethambutol vs. clarithromycin plus ethambutol as therapy for Mycobacterium avium complex bacteremia in patients with human immunodeficiency virus infection. Veterans Affairs HIV Research Consortium. Clin Infect Dis 1998:27:1278-85.

27 Heidarieh P, Mirsaeidi M, Hashemzadeh M, et al. In Vitro antimicrobial susceptibility of nontuberculous mycobacteria in Iran. Microb Drug Resist 2016:22:172-8.

28 Van den Eede P, Van Wesenbeeck L, Verlinden Y, et al. HIV-1 genotyping of the protease-reverse transcriptase and integrase genes to detect mutations that confer antiretroviral resistance. Methods Mol Biol 2013;1030:37-55.

29 Salvaña EMT, Schwem BE, Ching PR, et al. The changing molecular epidemiology of HIV in the Philippines. Int J Infect Dis 2017;61:44-50.

30 Chen Y, Hora B, DeMarco T, et al. Increased predominance of HIV-1 CRF01_AE and its recombinants in the Philippines. J Gen Virol 2019;100:511-22.

31 Taylor BS, Sobieszczyk ME, McCutchan FE, et al. The challenge of HIV-1 subtype diversity. N Engl J Med 2008;358:1590-602.

32 Costello C, Nelson KE, Suriyanon V, et al. HIV-1 subtype E progression among northern Thai couples: traditional and non-traditional predictors of survival. Int J Epidemiol 2005:34:577-84.

33 Kityo C Szubert AJ, Siika A et al. Raltegravir-intensified initial antiretrovira therapy in advanced HIV disease in Africa: A randomised controlled trial. PLoS Med 2018;15:1-20.

34 Raffi F, Rachlis A, Stellbrink HJ, et al. Once-daily dolutegravir versus raltegravir in antiretroviral-naive adults with HIV-1 infection: 48 week results from the randomised, double-blind, non-inferiority SPRING-2 study. Lancet 2013:381:735-43.

35 Rutstein SE, Chen JS, Nelson JAE, et al. High rates of transmitted NNRTI resistance among persons with acute HIV infection in Malawi: implications for first-line dolutegravir scale-up. AIDS Res Ther 2019;16:5 
36 Karade S, Sen S, Sashindran VK, et al. Absence of Integrase Strand Transfer Inhibitor Associated Resistance in Antiretroviral Therapy Naïve and Experienced Individuals from Western India. AIDS Res Hum Retroviruses 2019;0:1-10.

37 World Health Organization. HIV drug resistance report. 2017. http;//www.who.int/hiv/ pub/drugresistance/hivdr-report-2017/en/

38 Dong K, Ye L, Leng Y, et al. Prevalence of HIV-1 drug resistance among patients with antiretroviral therapy failure in Sichuan, China, 2010-2016. Tohoku J Exp Med 2019:247:1-12.

39 Ngo-Giang-Huong N, Huynh THK, Dagnra AY, et al. Prevalence of pretreatment HIV drug resistance in West African and Southeast Asian countries. J Antimicrob Chemother 2019;74:462-7.

40 Salvana EM, Dungca N, Arevalo G, et al. 1282. Detection of HIV Transmitted Drug Resistance by Next-Generation Sequencing in a CRF01_AE Predominant Epidemic. Open Forum Infect Dis 2018;5(Suppl 1):S391.

41 Gagliardini R, Ciccullo A, Borghetti A, et al. Impact of the M184V Resistance Mutation on Virological Efficacy and Durability of Lamivudine-Based Dual Antiretroviral
Regimens as Maintenance Therapy in Individuals with Suppressed HIV-1 RNA: A Cohort Study. Open Forum Infect Dis 2018;5:ofy113.

42 Kuehl AK, Noormohamed SE. Recombinant erythropoietin for zidovudine-induced anemia in AIDS. Ann Pharmacother 1995:29(7-8):778-9.

43 Henry DH, Beall GN, Benson CA, et al. Recombinant human erythropoietin in the treatment of anemia associated with human immunodeficiency virus (HIV) infection and zidovudine therapy. Overview of four clinical trials. Ann Intern Med 1992;117:739-48.

44 Tang MW, Shafer RW. HIV-1 antiretroviral resistance: scientific principles and clinica applications. Drugs 2012;72:e1-e25.

45 Hejazi N, Rajikan R, Choong CL, et al. Metabolic abnormalities in adult HIV infected population on antiretroviral medication in Malaysia: a cross-sectional survey. BMC Public Health 2013;13:758.

46 Raffi F, Esser S, Nunnari G, et al. Switching regimens in virologically suppressed HIV1-infected patients: evidence base and rationale for integrase strand transfer inhibitor (INSTI)-containing regimens. HIV Med 2016;17:3-16.

Copyright 2019 BMJ Publishing Group. All rights reserved. For permission to reuse any of this content visit https://www.bmj.com/company/products-services/rights-and-licensing/permissions/

BMJ Case Report Fellows may re-use this article for personal use and teaching without any further permission.

Become a Fellow of BMJ Case Reports today and you can:

- Submit as many cases as you like

- Enjoy fast sympathetic peer review and rapid publication of accepted articles

- Access all the published articles

Re-use any of the published material for personal use and teaching without further permission

\section{Customer Service}

If you have any further queries about your subscription, please contact our customer services team on +44 (0) 2071111105 or via email at support@bmj.com.

Visit casereports.bmj.com for more articles like this and to become a Fellow 